\section{La mortalidad por armas de fuego en Argentina entre 1990 y 2008}

\author{
Firearm mortality in Argentina, 1990-2008
}

\section{Abstract}

This is a descriptive epidemiological study of firearm mortality in Argentina, 1990-2008. The study compared the firearm mortality percentages and rates between the country's jurisdictions. The data are from the Office of Health Statistics and Information, Ministry of Health. From 1990 to 2008 there were 358,484 deaths from external causes, of which $16.6 \%(59,339)$ were caused by firearms. The Province of Buenos Aires showed the highest percentage and crude and adjusted rates. The jurisdictions in the Pampeana region (including the Province of Buenos Aires) showed a more homogeneous profile, similar to that of the country as a whole, with an increase in the rates from 1999 to 2002 and a decrease from 2003 to 2006. These findings are partially explained by the country's deteriorating socioeconomic conditions in the late 20th century, with a greater impact on Buenos Aires, one of the provinces of Argentina with the greatest development and economic dynamism. The situation in Buenos Aires was aggravated by the fact that the Province has one of the country's most repressive police forces.

Firearms; Violence; Mortality
Marina Gabriela Zunino 1

Edinilsa Ramos de Souza ${ }^{2}$

\section{Introducción}

Argentina, junto a Uruguay y Chile, registra las menores tasas de mortalidad por violencias entre los países de Latinoamérica; éstas se encuentran muy por debajo de las de México, Colombia, El Salvador y Brasil. Sin embargo, la problemática de la violencia social en Argentina se ha incrementado en los inicios del presente siglo.

Este trabajo constituye una ampliación del estudio sobre la situación de la mortalidad por armas de fuego en Argentina entre 1990 y 2005, cuestión que fue analizada en un artículo previo para su comparación con Brasil 1. En el análisis anterior se observó un aumento progresivo de las muertes por armas de fuego desde 1990 hasta 2002, año en que la tasa prácticamente se duplicó en relación a la de 1990. Los riesgos más altos se registraron en 2001 y 2002, con predominancia de los homicidios entre todas las intencionalidades.

El presente artículo tiene por objetivo analizar las diferencias en los porcentajes y las tasas de mortalidad por armas de fuego entre las jurisdicciones del país, entre 1990 y 2008. Se pretende así aportar el conocimiento de la situación de mortalidad por armas de fuego, lo que resulta innovador, considerando los escasos trabajos existentes sobre el tema, así como de aquellos que aborden el análisis de una serie temporal como la estudiada y de todas las jurisdicciones del país. 
Se discute la siguiente hipótesis: los resultados encontrados estarían explicados en parte por las políticas aplicadas en la última década del siglo XX en Argentina y que llevaron a una profunda crisis socioeconómica e institucional. La crisis, afectó a todo el país, pero habría tenido mayor impacto en algunas provincias de la Región Pampeana, en particular la Provincia de Buenos Aires. Esta situación habría estado agravada por el hecho de que dicha provincia tiene una de las fuerzas policiales más represivas del país.

\section{Material y métodos}

Este trabajo constituye un estudio epidemiológico descriptivo de la mortalidad por armas de fuego en Argentina durante 1990-2008.

Se analizó el número, porcentaje y tasas crudas y ajustadas por el método directo. La intencionalidad de las muertes se catalogó según la 9a y 10a Clasificación Internacional de Enfermedades y su equivalencia 2,3: accidentes por armas de fuego (E922; W32-W34), suicidios por armas de fuego (E955.0-E955.4; X72-X74), homicidios por armas de fuego (E965.0-E965.4; X93-X95) eventos de intención no determinada por armas de fuego (E970, E985.0-E985.4; Y22-Y24, Y35.0). Las muertes se caracterizaron según sexo y grupos de edad.

Para facilitar la descripción, los resultados de las jurisdicciones se presentan ordenados según regiones, aunque los datos no son aquí analizados a este nivel. La división por regiones utilizada corresponde a la del Instituto Nacional de Estadísticas y Censos (INDEC) 4: Región Cuyo (San Luis, San Juan y Mendoza); Región Noreste Argentino (Corrientes, Formosa, Chaco y Misiones); Región Noroeste Argentino (Catamarca, Jujuy, La Rioja, Tucumán, Salta y Santiago del Estero); Región Pampeana (Provincia de Buenos Aires, Ciudad de Buenos Aires, Córdoba, Entre Ríos, La Pampa y Santa Fe); y Región Patagónica (Chubut, Neuquén, Río Negro, Santa Cruz y Tierra del Fuego).

Las bases de datos provienen de los Informes Estadísticos de Defunción del Sistema de Estadísticas Vitales, proporcionadas por la Dirección de Estadística e Información en Salud (DEIS), Ministerio de Salud de la República Argentina.

En función de la Ley de Secreto Estadístico $17.622 / 68$, referida a la confidencialidad de la identidad de las personas que aparecen en las bases de datos oficiales de Argentina, las bases disponibilizadas por la DEIS no contienen ninguna información relativa a la identidad de los occisos.
Las proyecciones de población utilizadas corresponden a la serie de población "empalmada” entre los censos, que comprende el período 1990-2000. Esta serie fue proporcionada por el INDEC 5. Fue utilizada además la Serie Análisis Demográfico $N^{\circ} 31$ que continúa "la empalmada" con las estimaciones de población desde 20016 . Ambas series corresponden a la proyección de población al 30 de junio de cada año, según grupos etarios y sexos para el total país, Ciudad de Buenos Aires y provincias.

Dado el bajo número de muertes por armas de fuego en algunas de las provincias, las muertes a nivel de las jurisdicciones se analizaron en periodos de años agrupados. Se crearon cuatrienios (1991-1994, 1995-1998, 1999-2002, 2003-2006), teniendo en cuenta que los valores de mortalidad de 1991 y 2006 no difieren sustancialmente de los valores de 1990 y 2007 y 2008 respectivamente, por lo que el análisis de tendencia no se vería distorsionado.

Como referencia para el ajuste de tasas se utilizó la población total argentina, estimada para 2001. Los intervalos de confianza de las tasas crudas fueron calculados según el Washington State Department of Health 7 y los de las ajustadas corresponden a los calculados por el programa Epidat 3.0 (Xunta de Galicia, España; http:// dxsp.sergas.es/default.asp) durante el proceso de ajuste.

El análisis de los datos se realizó con el programa Excel 2000 (Microsoft Corp., Estados Unidos) y el georeferenciamiento con el MapInfo 7.0 (MapInfo Corp., New York, Estados Unidos).

\section{Resultados}

Entre 1990 y 2008 se produjeron en Argentina 358.484 muertes por causas externas, de las cuales el 16,6\% (59.339) correspondió a armas de fuego. La contribución de las armas de fuego a las causas externas aumentó progresivamente desde 1990 hasta 2002, pasó de 13,2\% a 22,3\%. Luego se registró un declive paulatino hasta 2008.

La tasa cruda de Argentina en el periodo 1990-2008 fue de 8,6 muertes por armas de fuego/100.000 habitantes. Las tasas anuales mostraron tendencia al aumento progresivo desde 1991 hasta 2002. Las tasas ajustadas más altas se registraron en 2001 y 2002, descendieron marcadamente hasta 2006 y se mantuvieron estables en 2007 y 2008 (6,2 muertes por armas de fuego/100.000 habitantes).

Como puede observarse en la Figura 1 y en la Tabla 1, los porcentajes y las tasas de mortalidad por armas de fuego mostraron grandes diferencias entre las jurisdicciones. La Provincia de 
Figura 1

Tasas ajustadas de muertes por armas de fuego (por 100.000 habitantes) según jurisdicción. Argentina, 1990-2008.
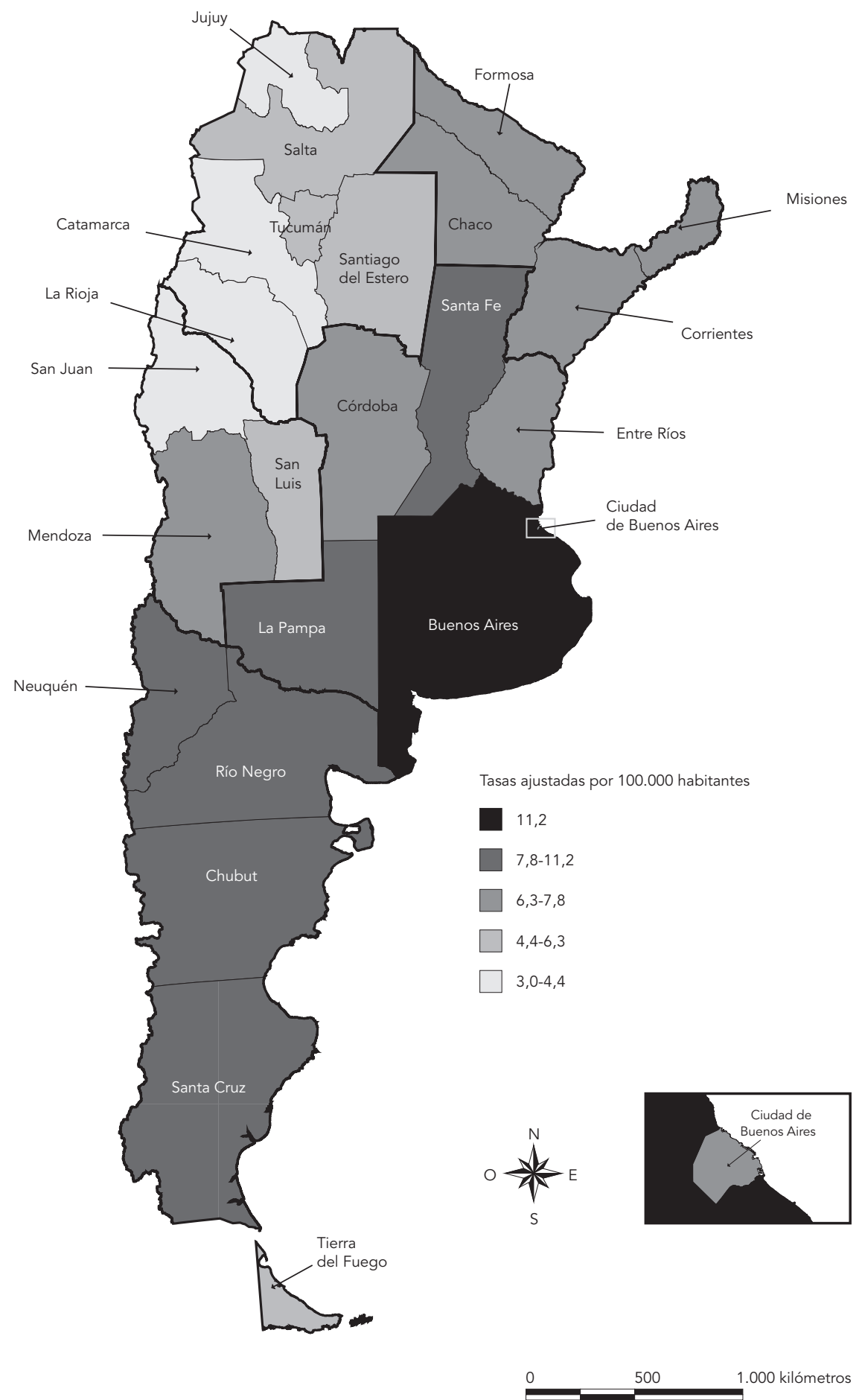

Fuente: Dirección de Estadística e Información en Salud, Ministerio de Salud e Instituto Nacional de Estadísticas y Censos. 
Número, porcentaje y tasas crudas y ajustadas (por 100.000 habitantes) de muertes por armas de fuego e intervalos de confianza (IC95\%) según región y jurisdicción. Argentina, 1990-2008.

\begin{tabular}{|c|c|c|c|c|c|c|}
\hline \multirow[t]{2}{*}{ Región/Jurisdicción } & \multirow[t]{2}{*}{$\mathrm{n}$} & \multirow[t]{2}{*}{$\%$} & \multicolumn{2}{|c|}{ Tasa cruda } & \multicolumn{2}{|c|}{ Tasa ajustada } \\
\hline & & & Tasa & IC95\% & Tasa & IC95\% \\
\hline \multicolumn{7}{|l|}{ Cuyo } \\
\hline Mendoza & 2.205 & 3,7 & 7,6 & $7,2-7,9$ & 7,5 & $7,2-7,8$ \\
\hline San Juan & 428 & 0,7 & 3,9 & $3,5-4,3$ & 3,8 & $3,5-4,2$ \\
\hline San Luis & 377 & 0,6 & 6,0 & $5,3-6,6$ & 5,9 & $5,3-6,5$ \\
\hline Total & 3.010 & 5,1 & 6,2 & $6,0-6,5$ & 6,7 & $6,5-7,0$ \\
\hline \multicolumn{7}{|l|}{ Noreste Argentino } \\
\hline Corrientes & 1.055 & 1,8 & 6,4 & $6,0-6,8$ & 6,6 & $6,2-7,0$ \\
\hline Chaco & 1.153 & 1,9 & 6,7 & $6,3-7,1$ & 6,9 & $6,4-7,3$ \\
\hline Formosa & 560 & 0,9 & 6,6 & $6,0-7,2$ & 7,0 & $6,4-7,6$ \\
\hline Misiones & 1.050 & 1,8 & 6,2 & $5,8-6,6$ & 6,5 & $6,1-6,9$ \\
\hline Total & 3.818 & 6,4 & 6,1 & $6,0-6,3$ & 7,0 & $6,8-7,3$ \\
\hline \multicolumn{7}{|l|}{ Noroeste Argentino } \\
\hline Catamarca & 176 & 0,3 & 3,0 & $2,6-3,5$ & 3,1 & $2,6-3,5$ \\
\hline Jujuy & 318 & 0,5 & 2,8 & $2,5-3,2$ & 3,0 & $2,7-3,3$ \\
\hline La Rioja & 185 & 0,3 & 3,7 & $3,1-4,2$ & 3,7 & $3,2-4,2$ \\
\hline Salta & 791 & 1,3 & 4,1 & $3,8-4,5$ & 4,4 & $4,1-4,7$ \\
\hline Santiago del Estero & 649 & 1,1 & 4,5 & $4,1-4,8$ & 4,7 & $4,3-5,1$ \\
\hline Tucumán & 1.311 & 2,2 & 5,5 & $5,2-5,8$ & 5,4 & $5,1-5,7$ \\
\hline Total & 3.430 & 5,8 & 4,2 & $4,0-4,3$ & 4,6 & $4,4-4,8$ \\
\hline \multicolumn{7}{|l|}{ Pampeana } \\
\hline Provincia de Buenos Aires & 30.118 & 50,8 & 12,0 & $11,9-12,2$ & 11,2 & $11,0-11,3$ \\
\hline Ciudad de Buenos Aires & 4.599 & 7,8 & 8,1 & $8,0-8,6$ & 7,2 & $7,0-7,5$ \\
\hline Córdoba & 4.270 & 7,2 & 7,6 & $7,4-7,8$ & 7,2 & $7,0-7,4$ \\
\hline Entre Ríos & 1.353 & 2,3 & 6,5 & $6,1-6,8$ & 6,3 & $6,0-6,7$ \\
\hline La Pampa & 481 & 0,8 & 8,9 & $8,1-9,8$ & 8,5 & $7,8-9,3$ \\
\hline Santa Fe & 4.713 & 7,9 & 8,2 & $7,9-8,4$ & 8,1 & $7,9-8,3$ \\
\hline Total & 45.534 & 76,7 & 9,8 & $9,7-9,9$ & 9,9 & $9,8-10,0$ \\
\hline \multicolumn{7}{|l|}{ Patagónica } \\
\hline Chubut & 708 & 1,2 & 9,0 & $8,3-9,8$ & 9,5 & $8,8-10,2$ \\
\hline Neuquén & 738 & 1,2 & 8,9 & $8,2-9,6$ & 8,6 & $8,0-9,3$ \\
\hline Río Negro & 789 & 1,3 & 7,6 & $7,0-8,2$ & 7,8 & $7,2-8,3$ \\
\hline Santa Cruz & 287 & 0,5 & 8,5 & $7,5-9,6$ & 8,8 & $7,7-9,9$ \\
\hline Tierra del Fuego & 94 & 0,2 & 5,5 & $4,4-6,9$ & 5,6 & $4,3-6,9$ \\
\hline Total & 2.616 & 4,4 & 8,0 & $7,7-8,3$ & 8,8 & $8,5-9,2$ \\
\hline \multicolumn{7}{|l|}{ Región desconocida } \\
\hline Jurisdicción desconocida & 931 & 1,6 & - & - & - & - \\
\hline Total regiones/Total jurisdicciones & 59.339 & 100,0 & 8,6 & $8,5-8,6$ & 8,6 & $8,5-8,6$ \\
\hline
\end{tabular}

Fuente: Dirección de Estadística e Información en Salud, Ministerio de Salud e Instituto Nacional de Estadísticas y Censos.

Buenos Aires, como era de esperar, dado que tiene la mayor cantidad de población de Argentina, concentró el 50,8\% de las muertes por armas de fuego del país entre 1990 y 2008. No obstante, también presentó las tasas cruda y ajustada más altas. Es esta provincia la que determina los valo- res superiores de muertes por armas de fuego de la Región Pampeana.

Después de Provincia de Buenos Aires, las tasas superiores se registraron en algunas provincias de la Región Patagónica. Debe considerarse, sin embargo, que estas provincias tienen 
bajo número de muertes por armas de fuego y de población, por lo que la magnitud de sus tasas obedecería a esta cuestión, más que al hecho de que el riesgo de morir sea alto. Como se observa en la Tabla 1, las tasas de estas provincias tienen intervalos de confianza amplios (con diferencias entre los límites superior e inferior de 0,8 a 1,0), lo que indica una alta variabilidad y baja precisión en las magnitudes de sus tasas. Esto último es válido también para La Pampa, para San Juan y San Luis (Región Cuyo), para todas las provincias del Noreste Argentino y para Catamarca y La Rioja del Noroeste Argentino.

Después de la Provincia de Buenos Aires y con excepción de las provincias recién mencionadas, Santa Fe, Ciudad de Buenos Aires y Córdoba registraron los pesos y tasas ajustadas de muertes por armas de fuego más altos de la serie analizada. A pesar de que el peso de las muertes por armas de fuego en Mendoza fue bajo, las tasas de esta provincia fueron también altas.

Resulta difícil concluir acerca de la evolución de las tasas de mortalidad por armas de fuego, ya que se observa gran variación en los resultados de las distintas jurisdicciones. Dada la similitud entre las tasas crudas y ajustadas, en la Tabla 2 se presentan sólo las ajustadas. Además, por ambos hechos (alta variabilidad y similitud entre valores de las tasas), para el análisis de tendencia se estudiaron también las tasas específicas por grupos etarios entre los cuatrienios; pero por cuestiones de espacio no se presentan los resultados de las tasas específicas. Considerando el grupo con mayor riesgo de muertes por armas de fuego en Argentina (el de 20 a 29 años que concentró el $26 \%$ de las muertes por armas de fuego en el total de la serie estudiada), en ocho de las veinticuatro jurisdicciones se observó un aumento desde el primer cuatrienio hasta 1999-2002 y luego una disminución en 2003-2006; un perfil similar al del país. Entre las ocho jurisdicciones con este perfil de muertes por armas de fuego se encuentran Ciudad de Buenos Aires, Provincia de Buenos Aires, Mendoza y Santa Fe, así como Entre Ríos, Chaco, Formosa y Neuquén. Esta tendencia se puede observar también para el total de muertes por armas de fuego según jurisdicción en las provincias de la Región Pampeana, Mendoza y Chaco (Tabla 2).

Como era de esperar, las armas de fuego tuvieron mayor peso entre los homicidios que entre el resto de las intencionalidades de muerte. En todo el período el 52,4\% (19.107) de los homicidios involucró armas de fuego, mientras que entre los suicidios el $34 \%$ (17.130), entre las muertes de intención no determinada el 27,3\% (19.856) y entre los accidentes el 1,6\% (3.246).

Dada la heterogeneidad presentada entre las jurisdicciones en cuanto a las intencionalidades de muerte, se hace también difícil sintetizar estos resultados. Sumado a esto está el hecho de que en muchos casos los números de muertes son aún más pequeños cuando se distribuyen según intencionalidad.

De manera sintética, resaltamos que los mayores riesgos de morir por homicidio por armas de fuego se registraron en Mendoza, Provincia de Buenos Aires y Misiones; esto sin considerar a Neuquén, Chubut y Formosa, en las que si bien el número de homicidios por armas de fuego no se considera pequeño, sus intervalos de confianza tienen un rango amplio, cercano a la unidad, lo que indica poca precisión en el cálculo de sus tasas (Tabla 3). Del análisis de tendencia resultó que en once de las veinticuatro jurisdicciones, incluidas Ciudad de Buenos Aires, Córdoba, Mendoza, Provincia de Buenos Aires y Santa Fe, las tasas ajustadas de homicidios por armas de fuego aumentaron hasta 1999-2002 y disminuyeron en 2003-2006; perfil similar al del país. El resto de las provincias mostraron perfiles variables $y$ disímiles al del país.

A excepción de Formosa, La Pampa, San Luis y las provincias de la Patagonia, cuyos intervalos de confianza fueron amplios, las tasas ajustadas de suicidios por armas de fuego más altas correspondieron a Mendoza (Cuyo), Entre Ríos, Provincia de Buenos Aires, Santa Fe (Pampeana), Corrientes, Chaco (Noreste Argentino) y Ciudad de Buenos Aires. No se encontró un patrón homogéneo de suicidios por armas de fuego de las jurisdicciones con respecto al perfil del país. En ocho de las veinticuatro jurisdicciones se registraron las tasas ajustadas de suicidios por armas de fuego más altas en 1991-1994; lo que incluyó a Provincia de Buenos Aires y Ciudad de Buenos Aires. En Mendoza el aumento se registró en 1995-1998 y en Santa Fe en 1999-2002.

En cuanto a los accidentes con armas de fuego Córdoba, Santa Fe y Corrientes presentaron los mayores riesgos. El perfil de accidentes con armas de fuego de Córdoba y Santa Fe coincidió con el de Argentina, un incremento hasta 19992002 y disminución en 2003-2006.

Dadas las características del grupo de muertes de intención no determinada por armas de fuego, resulta más relevante que describir las tasas, analizar el peso que éstas representan en relación al total de muertes en cada jurisdicción. Entre las provincias con mayor porcentaje de muertes de intención no determinada por armas de fuego destacan Santiago del Estero (45,6\%), Tucumán (43,1\%) y Provincia de Buenos Aires $(44,9 \%)$. A éstas le siguen San Juan, Córdoba, Jujuy, Entre Ríos y Ciudad de Buenos Aires, con pesos de entre $23,9 \%$ y $34,1 \%$. A excepción de Córdoba, estos porcentajes fueron inferiores a los de homicidios 
Tasas ajustadas de muertes por armas de fuego (por 100.000 habitantes) e intervalos de confianza (IC95\%) según región y jurisdicción. Argentina, 1991-1994, 1995-1998, 1999-2002 y 2003-2006 *.

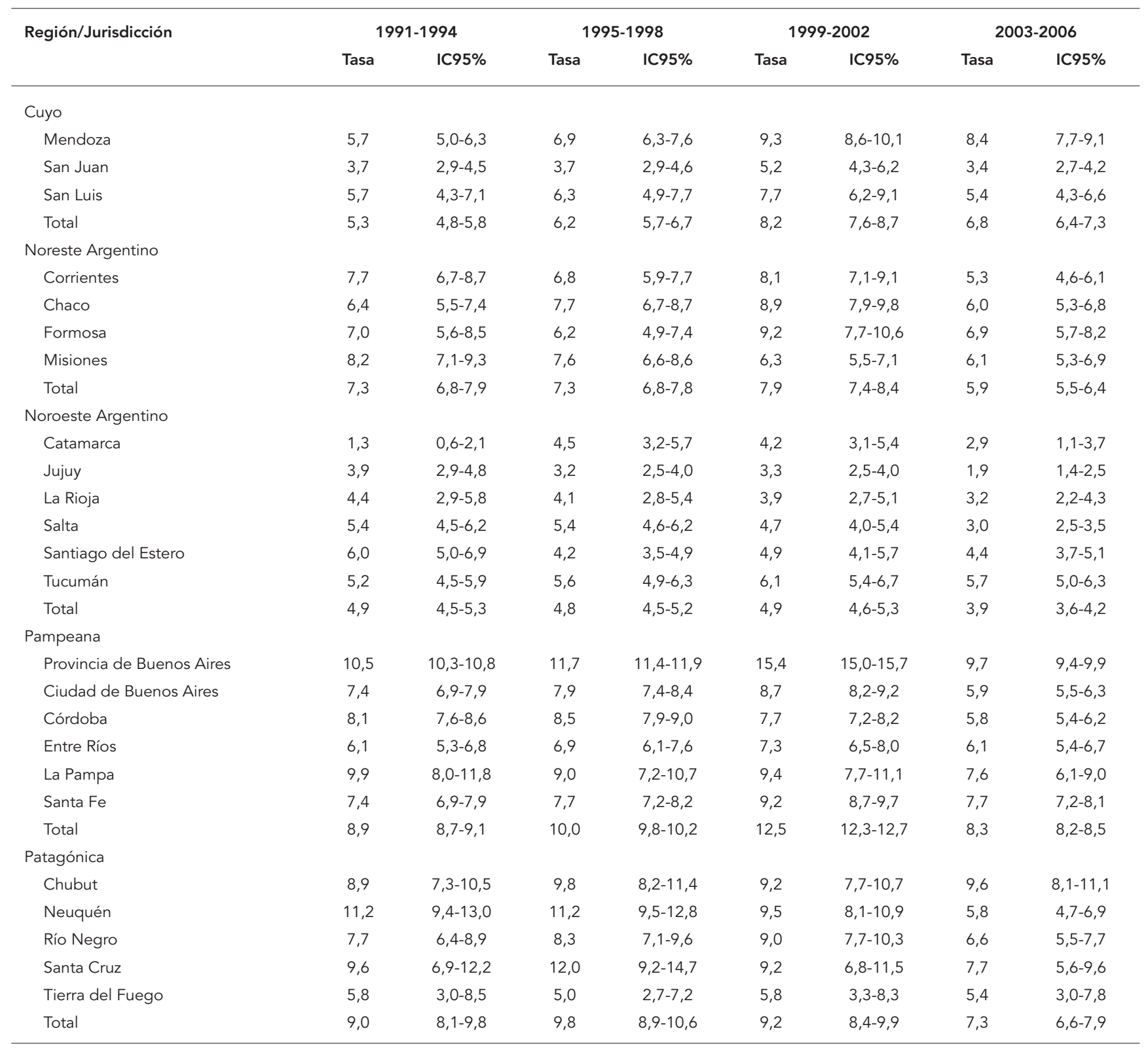

* La diferencia numérica observada en los totales de esta tabla con los de la Tabla 1 se debe a que estos valores corresponden al total del período 1991-2006, pues en la agrupación en cuatrienios se omitieron los años 1990, 2007 y 2008.

Fuente: Dirección de Estadística e Información en Salud, Ministerio de Salud e Instituto Nacional de Estadísticas y Censos.

y suicidios en las respectivas jurisdicciones. El peso de las muertes de intención no determinada por armas de fuego en Salta, Santa Fe, Tierra del Fuego y Chaco se registró entre 17,9 y 19,0. El porcentaje de muertes de intención no determinada por armas de fuego en el resto de las provincias varió entre $2 \%$ y $16,1 \%$.
Finalmente, en 1990-2008, el 19,3\% (50.861) de las muertes por causas externas en varones involucró armas de fuego y el restante $80,7 \%$ correspondió a muertes que involucraron otros mecanismos. Entre las mujeres las muertes por armas de fuego se registraron en el 8,6\% (7.883) de las defunciones por causas externas. Las muertes 
Porcentaje y tasas ajustadas de muertes por armas de fuego (por 100.000 habitantes) e intervalos de confianza (IC95\%) según intencionalidad. Argentina, 1990-2008.

\begin{tabular}{|c|c|c|c|c|c|c|c|c|c|c|c|c|}
\hline \multirow[t]{2}{*}{ Región/Jurisdicción } & \multicolumn{3}{|c|}{$\begin{array}{c}\text { Accidentes por armas } \\
\text { de fuego }\end{array}$} & \multicolumn{3}{|c|}{$\begin{array}{c}\text { Suicidios por armas } \\
\text { de fuego }\end{array}$} & \multicolumn{3}{|c|}{$\begin{array}{l}\text { Homicidios por armas } \\
\text { de fuego }\end{array}$} & \multicolumn{3}{|c|}{$\begin{array}{c}\text { Muertes de intención } \\
\text { no determinada por armas } \\
\text { de fuego }\end{array}$} \\
\hline & $\%$ & Tasa & IC95\% & $\%$ & Tasa & IC95\% & $\%$ & Tasa & IC95\% & $\%$ & Tasa & IC95\% \\
\hline \multicolumn{13}{|l|}{ Cuyo } \\
\hline Mendoza & 1,8 & 0,1 & $0,0-0,2$ & 35,4 & 2,7 & $2,5-2,9$ & 60,9 & 4,6 & $4,3-4,8$ & 2,0 & 0,1 & $0,1-0,2$ \\
\hline San Juan & 3,3 & 0,1 & $0,0-0,2$ & 25,2 & 1,0 & $0,8-1,2$ & 37,4 & 1,4 & $1,2-1,6$ & 34,1 & 1,3 & $1,1-1,5$ \\
\hline San Luis & 6,9 & 0,4 & $0,2-0,5$ & 56,0 & 3,4 & $2,9-3,8$ & 32,6 & 1,9 & $1,5-2,2$ & 4,5 & 0,3 & $0,1-0,4$ \\
\hline Total & 2,7 & 0,2 & $0,1-0,2$ & 36,5 & 2,4 & $2,3-2,5$ & 54,0 & 3,4 & $3,2-3,6$ & 6,8 & 0,4 & $0,4-0,5$ \\
\hline \multicolumn{13}{|l|}{ Noreste Argentino } \\
\hline Corrientes & 12,7 & 0,8 & $0,6-0,9$ & 37,1 & 2,5 & $2,3-2,8$ & 36,3 & 2,4 & $2,1-2,6$ & 13,9 & 0,9 & $0,8-1,1$ \\
\hline Chaco & 8,0 & 0,5 & $0,4-0,6$ & 36,2 & 2,6 & $2,3-2,9$ & 38,0 & 2,5 & $2,3-2,7$ & 17,9 & 1,2 & $1,1-1,4$ \\
\hline Formosa & 10,5 & 0,7 & $0,5-0,8$ & 37,1 & 2,6 & $2,2-3,0$ & 42,9 & 3,0 & $2,6-3,4$ & 9,5 & 0,7 & $0,5-0,8$ \\
\hline Misiones & 11,8 & 0,7 & $0,6-0,8$ & 22,0 & 1,6 & $1,4-1,8$ & 51,4 & 3,3 & $3,0-3,6$ & 14,8 & 0,9 & $0,8-1,1$ \\
\hline Total & 10,7 & 0,7 & $0,6-0,7$ & 32,7 & 2,3 & $2,2-2,5$ & 41,9 & 2,8 & $2,6-2,9$ & 14,7 & 1,0 & $0,9-1,1$ \\
\hline \multicolumn{13}{|l|}{ Noroeste Argentino } \\
\hline Catamarca & 5,1 & 0,2 & $0,1-0,3$ & 62,5 & 2,0 & $1,6-2,4$ & 27,3 & 0,8 & $0,6-1,0$ & 5,1 & 0,1 & $0,1-0,2$ \\
\hline Jujuy & 13,5 & 0,4 & $0,3-0,5$ & 36,5 & 1,1 & $0,9-1,3$ & 20,8 & 0,6 & $0,5-0,8$ & 29,2 & 0,9 & $0,7-1,0$ \\
\hline La Rioja & 17,3 & 0,6 & $0,4-0,8$ & 46,5 & 1,8 & $1,4-2,2$ & 21,6 & 0,8 & $0,5-1,0$ & 14,6 & 0,5 & $0,3-0,7$ \\
\hline Salta & 11,5 & 0,4 & $0,4-0,5$ & 39,8 & 1,8 & $1,6-2,0$ & 29,7 & 1,3 & $1,1-1,5$ & 19,0 & 0,8 & $0,7-1,0$ \\
\hline Santiago del Estero & 11,9 & 0,5 & $0,4-0,6$ & 24,3 & 1,2 & $1,0-1,4$ & 18,2 & 0,9 & $0,7-1,0$ & 45,6 & 2,1 & $1,9-2,4$ \\
\hline Tucumán & 2,6 & 0,1 & $0,1-0,2$ & 25,0 & 1,4 & $1,2-1,5$ & 29,3 & 1,5 & $1,4-1,7$ & 43,1 & 2,3 & $2,1-2,5$ \\
\hline Total & 8,3 & 0,3 & $0,3-0,4$ & 32,4 & 1,5 & $1,4-1,6$ & 26,1 & 1,1 & $1,1-1,2$ & 33,2 & 1,5 & $1,4-1,5$ \\
\hline \multicolumn{13}{|l|}{ Pampeana } \\
\hline Provincia de Buenos Aires & 1,1 & 0,1 & $0,1-0,1$ & 23,3 & 2,6 & $2,5-2,7$ & 30,6 & 3,5 & $3,4-3,5$ & 44,9 & 5,0 & $4,9-5,1$ \\
\hline Ciudad de Buenos Aires & 0,7 & 0,1 & $0,0-0,1$ & 38,1 & 2,5 & $2,3-2,6$ & 37,3 & 2,9 & $2,8-3,1$ & 23,9 & 1,8 & $1,7-1,9$ \\
\hline Córdoba & 28,4 & 2,1 & $1,9-2,2$ & 26,7 & 1,9 & $1,8-2,0$ & 14,9 & 1,1 & $1,0-1,2$ & 30,0 & 2,2 & $2,1-2,3$ \\
\hline Entre Ríos & 5,9 & 0,4 & $0,3-0,4$ & 41,7 & 2,7 & $2,4-2,9$ & 24,5 & 1,6 & $1,4-1,7$ & 27,9 & 1,8 & $1,6-1,9$ \\
\hline La Pampa & 5,6 & 0,5 & $0,3-0,7$ & 75,1 & 6,4 & $5,7-7,1$ & 16,2 & 1,4 & $1,1-1,7$ & 3,1 & 0,3 & $0,1-0,4$ \\
\hline Santa Fe & 13,1 & 1,1 & $1,0-1,2$ & 33,7 & 2,6 & $2,5-2,8$ & 34,3 & 2,8 & $2,7-3,0$ & 18,9 & 1,5 & $1,4-1,6$ \\
\hline Total & 5,0 & 0,5 & $0,5-0,5$ & 27,3 & 2,5 & $2,5-2,6$ & 29,9 & 2,9 & $2,8-2,9$ & 37,8 & 3,6 & $3,6-3,7$ \\
\hline \multicolumn{13}{|l|}{ Patagónica } \\
\hline Chubut & 5,2 & 0,5 & $0,3-0,6$ & 40,7 & 4,1 & $3,6-4,5$ & 38,0 & 3,4 & $3,0-3,9$ & 16,1 & 1,5 & $1,2-1,8$ \\
\hline Neuquén & 4,7 & 0,4 & $0,2-0,5$ & 34,1 & 3,3 & $2,8-3,7$ & 48,6 & 4,0 & $3,5-4,4$ & 12,5 & 1,0 & $0,8-1,2$ \\
\hline Río Negro & 6,1 & 0,4 & $0,3-0,6$ & 43,2 & 3,5 & $3,1-3,9$ & 35,2 & 2,6 & $2,3-2,9$ & 15,5 & 1,2 & $1,0-1,4$ \\
\hline Santa Cruz & 2,8 & 0,2 & $0,1-0,4$ & 58,9 & 5,4 & $4,5-6,2$ & 27,9 & 2,4 & $1,8-2,9$ & 10,5 & 0,9 & $0,5-1,2$ \\
\hline Tierra del Fuego & 4,3 & 0,2 & $0,0-0,5$ & 47,9 & 2,6 & $1,8-3,5$ & 29,8 & 1,8 & $1,0-2,6$ & 18,1 & 1,0 & $0,5-1,4$ \\
\hline Total & 5,0 & 0,4 & $0,3-0,5$ & 41,9 & 3,8 & $3,5-4,0$ & 38,8 & 3,1 & $2,9-3,3$ & 14,3 & 1,2 & $1,1-1,3$ \\
\hline \multicolumn{13}{|l|}{ Región desconocida } \\
\hline Jurisdicción desconocida & 3,3 & - & - & 14,9 & - & - & 40,7 & - & - & 41,0 & - & - \\
\hline Total Regiones/ & 5,5 & 0,5 & $0,5-0,5$ & 28,9 & 2,5 & $2,7-2,8$ & 32,2 & 2,8 & $2,7-2,8$ & 33,5 & 2,9 & $2,8-2,9$ \\
\hline Total Jurisdicciones & & & & & & & & & & & & \\
\hline
\end{tabular}

Fuente: Dirección de Estadística e Información en Salud, Ministerio de Salud e Instituto Nacional de Estadísticas y Censos.

por armas de fuego predominaron en varones, con tasas superiores a las de mujeres en todas las jurisdicciones y en todos los cuatrienios analizados. El riesgo de muertes por armas de fuego en varones fue superior en 1999-2002, con una tasa de 15,8/100.000, y en mujeres fue en 1991-1994 con $2,6 / 100.000$. 
La tasa de muertes por armas de fuego en el grupo con mayor riesgo de muertes por armas de fuego (20-29 años) fue de 14,1/ 100.000 entre 1990 y 2008. Nuevamente, exceptuando a las provincias de Patagonia, resaltan Provincia de Buenos Aires, Mendoza y Santa Fe con tasas que superaron a la del país en dicho intervalo etario.

\section{Discusión}

Como síntesis de los resultados, puede decirse que la Provincia de Buenos Aires y Mendoza presentaron las mayores tasas del total de muertes por armas de fuego y de las distintas intencionalidades, especialmente de suicidios y homicidios por armas de fuego; esto con excepción de las provincias con bajo número de muertes y/o población. Fue en las provincias de la Región Pampeana donde se encontró un perfil más homogéneo y similar al del país para el total de muertes por armas de fuego, así como para las intencionalidades de muerte. Entre los cuatrienios se observó aumento de la mortalidad por armas de fuego en 1999-2002 y disminución en 2003-2006; excepto para los suicidios por armas de fuego que mostraron perfiles heterogéneos entre las provincias.

Entre los resultados destacaron también Chaco, Corrientes, Misiones y Tucumán. No obstante, estas provincias presentaron menores tasas totales de muertes por armas de fuego y de las distintas intencionalidades; a excepción de Tucumán que tuvo, junto a Santiago del Estero, una tasa y porcentaje de muertes de intención no determinada por armas de fuego superior incluso a algunas de las jurisdicciones de la Región Pampeana.

Por otra parte resaltamos que, a pesar de la alta magnitud de muertes de intención no determinada por armas de fuego en el total del período, éstas se registraron en porcentajes superiores al $30 \%$ en sólo tres de las veinticuatro jurisdicciones del país. Argentina, al igual que Brasil, entre otros países de Latinoamérica, está realizando esfuerzos por mejorar el sistema de información sobre mortalidad, cuestión que se hace evidente en la disminución del número de muertes de intención no determinada por armas de fuego a lo largo del período estudiado. Sería igualmente pertinente explorar las diferencias regionales, también en cuanto a la calidad de cumplimentación de los registros, a fin de descartar diferencias en la magnitud del subregistro entre las jurisdicciones; cuestión que no ha sido estudiada en Argentina.

En el caso de las provincias con baja cantidad de muertes por armas de fuego y/o de po- blación, pequeños cambios en el número de muertes pueden representar altas variaciones en el valor de las tasas. En función del análisis de la amplitud de los intervalos de confianza, así como del número de muertes y de las tasas específicas por edad según cuatrienios, podemos aceptar que esto ocurrió en La Pampa, San Juan y San Luis (Cuyo), todas las provincias del Noreste Argentino, Catamarca y La Rioja (Noroeste Argentino) y todas las provincias de la Región Patagónica. En todos estos casos, una menor variabilidad podría obtenerse con una agrupación en periodos mayores, sin embargo, se decidió conservar la agrupación en cuatrienios para posibilitar la comparación de la tendencia temporal (tasas ajustadas) entre las jurisdicciones de mayor número de muertes por armas de fuego. Cabe resaltar que son las tasas crudas las que muestran los riesgos de muerte reales observados para las provincias.

La heterogeneidad en los perfiles de mortalidad por violencias entre jurisdicciones es un resultado observado también en otros países. En Brasil, donde existen numerosos trabajos epidemiológicos al respecto, dicha heterogeneidad se explica por diferentes cuestiones, principalmente la socioeconómica y la urbanización, pues las muertes por violencias se concentran mayormente en las grandes metrópolis 8,9,10,11. El 90\% del territorio argentino es urbano ${ }^{4}$, con lo cual, y tal como será aquí desarrollado, la explicación a dichas divergencias las encontramos en cuestiones sociohistóricas y también socioeconómicas, más que en el grado de urbanización propiamente dicho.

Las preguntas que guían esta discusión son ¿Por qué en las jurisdicciones de la Región Pampeana, Provincia de Buenos Aires en particular, y por qué en el periodo 1999-2002 las muertes por armas de fuego tuvieron mayor importancia? ¿A qué se deben las diferencias en las tasas específicas según intencionalidad entre las jurisdicciones?

El hecho de que sea en dicha región y en el cuatrienio mencionado en el cual se presentaron las más altas tasas de mortalidad por armas de fuego nos remite nuevamente (como lo hicimos en Zunino et al. 1) a los acontecimientos ocurridos en la década de los 90, que como es obvio se asentaron en las bases históricas del país, y que llevaron a Argentina una gran crisis socioeconómica e institucional que hizo explosión a fines del 2000. El perfil observado de muertes según intencionalidad, en especial de los homicidios por armas de fuego y las muertes de intención no determinada por armas de fuego, podría asimismo estar relacionado con aspectos que llevaron a la crisis y a los acontecimientos ocurridos 
durante el momento más crítico (los años 2001 y 2002).

Basados en los documentos del Centro de Estudios Legales y Sociales 12, hemos explicado la idea de una relación entre la violencia y la última crisis socioeconómica e institucional vivida en Argentina 1. Durante 1999-2002 se registró un aumento de los delitos "comunes", que suponemos consecuencia de la necesidad de cubrir aspectos básicos de la vida que los niveles críticos de desempleo y la baja de salarios impedía cubrir. Principalmente en 2001 se observó un aumento de la violencia social relativa a las protestas callejeras de la población ante los efectos del deterioro socioeconómico y la falta de políticas de Estado que ayudaran a superar la situación. En respuesta a estas protestas se detectó un uso abusivo de la violencia institucional ejercida por la policía que produjo muertes y gran cantidad de heridos.

La hipótesis que formulamos es que los hallazgos de este trabajo son, en parte, consecuencia del deterioro socioeconómico. Planteamos que, si bien este deterioro afectó a todo el país, habría tenido mayor impacto en las jurisdicciones de la Región Pampeana, particularmente en Provincia de Buenos Aires, por ser ésta una de las provincias con mayor desarrollo y dinamismo económico de Argentina. Por otra parte, en Provincia de Buenos Aires la situación habría estado agravada por el hecho de que esta provincia tiene una de las fuerzas policiales más represivas del país. A continuación desarrollamos esta hipótesis exponiendo en primer lugar las ideas relativas a las cuestiones socioeconómicas y en segundo a las fuerzas policiales.

Aclaramos al margen que es nuestra intención que las cuestiones aquí presentadas ayuden a comprender el porqué de las diferencias observadas en cuanto a tasas de mortalidad por armas de fuego entre jurisdicciones y periodos, pero no intentamos dar una explicación acabada de estos hallazgos, ni sugerir que los dos asuntos tratados son los únicos que explican los perfiles observados de muertes por armas de fuego.

En una breve síntesis resaltamos que las transformaciones socioeconómicas realizadas entre 1990 y 2001 se basaron principalmente en la desregulación de la economía y la promoción y apertura al libre movimiento de capitales, en la privatización de la mayoría de las empresas públicas que prestaban servicios de uso económico masivo, y en la flexibilización, apertura y remoción de las regulaciones de la tradicional "red de seguridad laboral" que caracterizó al Estado de bienestar en Argentina ${ }^{12}$. En relación con las reformas laborales, los procesos de desregulación y flexibilización de las relaciones y condiciones de trabajo fueron los ejes centrales. Para mencionar sólo algunas, se crearon distintas modalidades de contratación laboral "promovidas" mediante rebajas o eliminación de cargas sociales, modificando así el concepto de relación laboral ininterrumpida y eximiendo a los empleadores de hasta el 50\% de su contribución al sistema de seguridad social; se definieron cambios en los métodos de ajuste salarial, promoviendo cláusulas del tipo "ajuste por productividad"; y se crearon los programas de empleo para los llamados "grupos especiales de trabajadores/as" que se ejecutaron desde 1993 12. Las modalidades precarias de contratación reemplazaron progresivamente a las relaciones formales, y se sumaron al crecimiento sostenido del empleo en negro y las relaciones propias del empleo informal. El desempleo, el subempleo y el empleo precario afectaron para 2002 a cerca de la mitad de la población económicamente activa.

Así, entre los principales cambios producidos en los ‘90 se encuentran el creciente nivel de desempleo, que afectó mayormente a los varones jóvenes, el debilitamiento y hasta destrucción de las organizaciones laborales, la depresión de los salarios y el aumento consecuente de la pobreza 13 .

Estas cuestiones podemos verlas reflejadas sólo en algunos indicadores socioeconómicos, lo que muestra la complejidad de los asuntos tratados. En 2001, la Ciudad de Buenos Aires presentó el menor porcentaje de necesidades básicas insatisfechas; las provincias de la regiones Pampeana, Patagónica y Cuyo presentaron también bajos porcentajes de dicho indicador, entre $10,3 \%$ y $17,9 \%$. Por el contrario, en las provincias del Noreste Argentino y del Noroeste Argentino el porcentaje de necesidades básicas insatisfechas varió entre 20,4\% y 33,6\% 14. Habría así una relación inversa entre las tasas de muertes por armas de fuego y el porcentaje de básicas insatisfechas. En cuanto a los indicadores de educación, las menores tasas de analfabetismo y los mayores porcentajes de población con nivel medio completo correspondieron también a Ciudad de Buenos Aires y a las provincias de las regiones Pampeana y Patagónica. Las provincias del Noreste Argentino presentaron las tasas de analfabetismo superiores y los niveles de educación media completa inferiores. En las provincias del Noroeste Argentino hubo gran variación en estos indicadores pero destacó Santiago del Estero con los peores niveles de educación.

Llama la atención que a pesar de la crisis estos indicadores mejoraron en todas las jurisdicciones entre los periodos censales (1991 y 2001) 14,15. Sin embargo, cuando se analizan las tasas de desocupación y pobreza se observa el resultado 
opuesto. Excepto en Santa Cruz, la desocupación se incrementó entre los censos. Las provincias de la Región Pampeana junto a Corrientes (Noreste Argentino), Catamarca, Jujuy y Santiago del Estero (Noroeste Argentino) presentaron el mayor incremento en las tasas de desocupación 16. Por otro lado, los incrementos superiores en el porcentaje de población bajo la línea de pobreza se registraron en las provincias de la Región Pampeana y en Mendoza.

En resumen, las provincias de la Región Patagónica tienen los mejores indicadores de necesidades básicas insatisfechas y educación mientras que el Noreste Argentino y el Noroeste Argentino tienen los peores. El aumento de la desocupación y la pobreza ocurrió en mayor medida en las provincias de la Región Pampeana, junto a algunas jurisdicciones del Noreste Argentino y Noroeste Argentino.

Una explicación a la aparente discordancia entre los indicadores de necesidades básicas insatisfechas y educación vs. los de desocupación y pobreza es que la crisis tuvo un impacto coyuntural que afectó directamente los niveles de empleo, y conllevó a su vez a un aumento de la pobreza, pero que no alcanzó a producir un deterioro a nivel estructural, que sí se vería reflejado en los niveles de educación, por ejemplo. Desde el 2003, las políticas de gobierno impulsan fuertemente el desarrollo de la economía y se logra generar un aumento del empleo y disminución de la pobreza con lo cual la situación coyuntural se revierte y se evita así la profundización del deterioro socioeconómico.

A partir de lo expuesto, si bien es claro que no existe relación directa entre condición socioeconómica y mortalidad por violencias, pues las jurisdicciones con menores porcentajes de necesidades básicas insatisfechas y niveles de educación tuvieron las mayores tasas de mortalidad por armas de fuego, podemos aceptar que en Argentina los efectos del brutal incremento en la desocupación y en los niveles de pobreza llevaron a un aumento de la violencia social, dada a través de los delitos "comunes", que muchas veces pueden acabar en homicidios generalmente cometidos con armas de fuego, y de los saqueos perpetuados para poder cubrir las necesidades básicas, así como de las manifestaciones callejeras para exigir al gobierno una mejoría de la situación.

Es lógico considerar que la violencia social se haya dado en mayor medida en la Región Pampeana, porque ésta incluye la zona central del país, zona que ha reunido tradicionalmente y con primacía hasta la actualidad al sector productivo de mayor desarrollo, que concentra la mayor cantidad de población trabajadora y cuya población está altamente sindicalizada; aspecto este último que permitió encontrar mecanismos de exigencia al gobierno para paliar la crisis $17,18,19$

El impacto de la crisis habría tenido menor fuerza en el Noreste y Noroeste Argentino, posiblemente porque en estas regiones existen condiciones estructurales de pobreza, desigualdad y desocupación que hicieron menos perceptible el deterioro producido. Esto reafirmaría la hipótesis de que no es la pobreza, sino la desigualdad, la que lleva a un incremento de los niveles de violencia. Es posible también que dadas las condiciones de deterioro estructural hayan existido en estas provincias dispositivos de redes sociales que permitieran resistir "mejor" los embates de la crisis, a la vez que posibilitaran un menor grado de violencia social. Estudios cualitativos deberían desarrollarse para responder estas hipótesis.

Por otra parte, en nuestra hipótesis planteamos el hecho de que las altas tasas de mortalidad por armas de fuego, particularmente los homicidios por armas de fuego y las muertes de intención no determinada por armas de fuego, registradas en toda la Región Pampeana, pero mayormente en Provincia de Buenos Aires en el periodo 1999-2002, sean el resultado del tradicional accionar represivo y muchas veces extralegal de las fuerzas policiales, que se puso en evidencia en su respuesta a la violencia social.

Como fuera mencionado en Zunino et al. 1, en base a datos de la Coordinadora Contra la Represión Policial e Institucional 20, entre noviembre del 2000 y noviembre de 2001 se mantuvo una tasa promedio de 10 personas asesinadas por mes por las fuerzas de seguridad y muchos de los casos que la versión policial presentó como enfrentamientos, la investigación judicial posterior reveló que fueron ejecuciones, enfrentamientos fraguados o circunstancias donde los policías utilizaron la fuerza sin respetar ninguno de los recaudos que establece la ley. Así, suponemos que gran parte de las muertes de intención no determinada por armas de fuego se corresponden a casos de homicidios por armas de fuego no especificados como tales. Demás está señalar la importante función que tuvieron las armas de fuego en el amedrentamiento y represión de la población. De hecho, el mayor número de autorizaciones de porte de armas de fuego en el país se registró mayoritariamente en las Fuerzas Policiales y Armadas, con valores superiores en 2001, 2003 y 2004 y posterior disminución. Si bien en menor magnitud, el porte de armas en la población civil también aumentó en los años cercanos a la crisis (2000-2003) y disminuyó paulatinamente hasta 200821. 
El accionar extralegal, que se evidenció claramente durante los peores años de la crisis, tiene explicación en el devenir histórico de las fuerzas policiales. Según Saín 22, a lo largo de la historia del país se estructuró un tipo de pacto en el que el gobierno central delegó a la policía la administración de la seguridad pública (la implementación de la política criminal, la conducción estratégica y operativa del propio cuerpo, etc.), y a cambio, la policía se comprometió a garantizarla. Como consecuencia, la mayoría de las agencias policiales federales y provinciales de Argentina tienen como rasgo distintivo la autonomía política frente al gobierno estatal y frente a la sociedad política y civil. Esta característica le permite desarrollar de forma autosustentada ciertas modalidades organizativas y de funcionamiento, y ciertas prácticas institucionales regulares. Así, el modelo policial tradicional ha supuesto el autogobierno sobre la seguridad pública y sobre el propio sistema policial. El autogobierno permitió garantizar un control politico-institucional interno con algunas prácticas ajustadas a las normas legales y reglamentarias y otras prácticas subterráneas o paralelas que se desarrollan al margen de las leyes y normas y que son "admitidas, impuestas, reguladas, gobernadas y rigurosamente controladas por la propia cúpula" 22 (p. 48)

Estas cuestiones son particularmente significativas en los dos cuerpos policiales más grandes del país: la Policía Federal Argentina y la Policía Bonaerense 22,23. La Policía Federal Argentina tiene jurisdicción exclusiva dentro del territorio de la Ciudad de Buenos Aires y competencia acotada a cuestiones federales en todo el territorio nacional; la Policía Bonaerense es la policía de la provincia de Buenos Aires.

Las fuerzas policiales argentinas tienen así un legajo histórico fortalecido durante la última dictadura militar (1976-1983) que dio pie a que se continuara en democracia con las prácticas abusivas. El panorama reciente se vuelve aún más complejo cuando, durante las últimas décadas, esta impronta de extralegalidad conllevó a la participación de algunos grupos de la policía en actividades ilícitas desarrolladas por organizaciones criminales. En este contexto, además de las prácticas tradicionales de violación a los derechos humanos, se institucionalizó, entre otras cuestiones, el tráfico de personas, drogas y armas. Sería particularmente en Provincia de Buenos Aires donde ha habido una enorme expansión de la criminalidad organizada, en la que está involucrada la Policía Bonaerense, y que ha llevado a niveles altísimos de violencias y ha sido una de las principales causas de homicidios dolosos 24 .
Las cuestiones expuestas en esta discusión corresponden principalmente a la Provincia de Buenos Aires. Fue sobre esta provincia que se encontró la mayor parte de las investigaciones académicas, posiblemente por la importancia económica y social de la misma y por las características particulares de su policía. Resaltamos por lo tanto la necesidad de ampliar los estudios para tener una mejor comprensión de los perfiles de muertes por armas de fuego y en general de las violencias en cada una de las jurisdicciones y de las diferencias observadas entre ellas.

Antes de finalizar hacemos mención a la disminución de las muertes por armas de fuego desde 2003. Esta disminución coincide con una mejoría de la situación socioeconómica en virtud del aumento del empleo y mejora de la calidad de vida. Hemos señalado también la idea de que las menores tasas se correspondan con el desarrollo de las campañas de desarme implementadas en el país y que permitieron disminuir su uso desde un 73,0\% en 2002 hasta un 60,7\% en 2005 25. Las armas de fuego son ya instrumentos de uso generalizado y fácil adquisición, y es sabido que su presencia aumenta la probabilidad de muerte 26 . Resulta entonces fundamental continuar con las iniciativas de desarme.

Acordamos con Kosovsky 27 en que la meta para reducir el circulante de armas de fuego y sus resultantes debe ser sólo parte de una política integral que atienda las diversas dimensiones sobre las que se asienta las violencias. En base a lo que señala este autor resaltamos que, si no se planifican en el marco de una estrategia global acciones tendientes a atacar fenómenos que operan en cada contexto social como motivadores de la violencia, entre ellos el desempleo, las desigualdades económicas y la segregación de algunos sectores de la sociedad, cualquier intento estatal que apunte a disminuir la demanda de armas de fuego y por ende la violencia producida estará destinada al fracaso.

Por otro lado, ha habido en los últimos años en Argentina un aumento de delitos y homicidios relacionados con el narcotráfico. En función de la sabida relación entre el desarrollo del narcotráfico y el contrabando y uso de armas de fuego resulta fundamental crear mecanismos estatales que frenen las actividades del crimen organizado. En este sentido, es de gran importancia continuar y mejorar la denominada por Saín 21 reforma policial "trunca”, así como implementar políticas que intenten deshacer las condiciones estructurales relativas a los problemas de seguridad, entre ellos el de consolidar una fuerza policial con personal altamente calificado en sus tareas, transparente, eficiente, con ciudadanos al servicio de la ciudadanía. 


\section{Resumen}

Este es un estudio epidemiológico descriptivo de la mortalidad por armas de fuego en Argentina, 1990-2008. Se analizaron los porcentajes y las tasas de mortalidad por armas de fuego entre las jurisdicciones del país. Los datos provienen de la Dirección de Estadística e Información en Salud, Ministerio de Salud y Ambiente, República Argentina. Entre 1990-2008 se produjeron 358.484 muertes por causas externas de las cuales el 16,6\% (59.339) correspondieron a armas de fuego. La Provincia de Buenos Aires presentó el porcentaje y tasas cruda y ajustada superiores. Las jurisdicciones de la Región Pampeana (incluye Provincia de Buenos Aires) presentaron un perfil más homogéneo y similar al del país: aumento de las tasas en 1999-2002 y disminución en 2003-2006. Estos hallazgos se explicarían en parte por el deterioro socioeconómico vivido en Argentina a fines del siglo XX, que habría tenido mayor impacto en Provincia de Buenos Aires, por ser ésta una de las provincias con mayor desarrollo y dinamismo económico de Argentina. La situación de Provincia de Buenos Aires habría estado agravada por el hecho de tener una de las fuerzas policiales más represivas del país.

Armas de Fuego; Violencia; Mortalidad

\section{Colaboradores}

M. G. Zunino realizó el procesamiento y análisis de los datos, así como la escritura del texto. E. R. Souza contribuyó con conceptos teóricos y escritura de la versión final del texto.

\section{Agradecimientos}

Este trabajo fue financiado por el Programa de Estudantes Convênio de Pós-Graduação (PEC-PG), Coordenação de Aperfeiçoamento de Pessoal de Nível Superior (CAPES), Brasil. Beca de doctorado No 03897-012006. Agradecemos la asesoría prestada por el Dr. Marcio Alazraqui y el Lic. Carlos Guevel; así como el apoyo recibido por el Dr. Hugo Spinelli y todo el equipo de la Maestría en Epidemiología, Gestión y Políticas de Salud y a la Universidad Nacional de Lanús.

\section{Referencias}

1. Zunino MG, Souza ER, Lauritzen B. Estudio epidemiológico comparativo de la mortalidad por armas de fuego en Brasil y Argentina, 1990-2005. Salud Colectiva 2008; 4:349-61.

2. Organización Panamericana de la Salud. Manual de la Clasificación Estadística Internacional de Enfermedades, Traumatismos y Causas de Defunción. Novena revisión. Washington DC: Organización Panamericana de la Salud; 1978.

3. Organización Panamericana de la Salud. Clasificación Estadística Internacional de Enfermedades y problemas relacionados con la salud, CIE-10. Washington DC: Organización Panamericana de la Salud; 1995. (Publicación Científica, 554).

4. Instituto Nacional de Estadística y Censos. Anuario estadístico de la República Argentina 2008. 2a Ed. Buenos Aires: Instituto Nacional de Estadística y Censos; 2010.
5. Instituto Nacional de Estadística y Censos. Estimaciones de población para el total país y provincias por sexo y grupos de edad 1990-2000. Buenos Aires: Ministerio de Economía y Producción de la Nación; 2009.

6. Instituto Nacional de Estadística y Censos. Proyecciones provinciales por sexo y grupos de edad 2001-2015. Buenos Aires: Instituto Nacional de Estadística y Censos; 2055. (Serie Análisis Demográfico, 31). http://www.indec.gov.ar/nuevaweb/ cuadros/2/proyecciones_provincialesvol31.pdf (accedido el 15/Ago/2010).

7. Washington State Department of Health. Guidelines for working with small numbers. Olympia: Washington State Department of Health; 2002. http://www.doh.wa.gov/data/guidelines/Small Numbers.htm (accedido el 20/Jul/2009). 
8. Nadanovsky P, Celeste RK, Wilson M, Daly M. Homicide and impunity: an ecological analysis at state level in Brazil. Rev Saúde Pública 2009; 43:733-42.

9. Souza ER, Lima MLC. The panorama of urban violence in Brazil and its capitals. Ciênc Saúde Coletiva 2006; 11:363-73.

10. Gawryszewski VP, Mello-Jorge MHP. Mortalidade violenta no Município de São Paulo nos últimos 40 anos. Rev Bras Epidemiol 2000; 1-3:50-69.

11. Mello-Jorge MHP. Análise dos dados de mortalidade. Rev Saúde Pública 1997; 31(4 Suppl):5-25.

12. Centro de Estudios Legales y Sociales. El Estado frente a la protesta social, 1996-2002. Buenos Aires: Siglo XXI Editores; 2003.

13. Romero D, Szwarcwald CL. Crisis económica y mortalidad infantil en Latinoamérica desde los años ochenta. Cad Saúde Pública 2000; 16: 799-814.

14. Instituto Nacional de Estadística y Censos. Censo nacional de población, hogares y viviendas del año 2001. Buenos Aires: Instituto Nacional de Estadística y Censos; 2001. http://www.indec.gov.ar/web censo/index.asp (accedido el 12/Jun/2010).

15. Instituto Nacional de Estadística y Censos. Situación y evolución social provincial. Síntesis provinciales, actualizaciones 2001-2002 [CD-ROM]. Buenos Aires: Ministerio de Economía y Producción de la Nación; 2006.

16. Cicowiez M. Caracterización económico social de las provincias Argentinas. La Plata: Departamento de Economía, Facultad de Ciencias Económicas, Universidad Nacional de La Plata; 2003. (Documento de Federalismo Fiscal, 5).

17. Centro de Estudios Legales y Sociales. Derechos humanos en Argentina. Informe 2007. Buenos Aires: Siglo XXI Editores; 2008.

18. Alazraqui M, Spinelli H, organizadores. Desigualdades en salud en el nivel local/municipal. Buenos Aires: Ediciones de la UNLa/Universidad Nacional de Lanús; 2008. (Colección Salud Comunitaria).

19. Malatesta AA. Notas para la historia de la industria argentina. Ensayo. http://www.edutecne.utn.edu. ar/historia-industria/hist-ind1.pdf (accedido el 02/May/2010).
20. Coordinadora Contra la Represión Policial e Institucional. Archivos de casos 1983-2001. Recopilación de muertes de personas a manos de las fuerzas de seguridad en Argentina. Buenos Aires: Coordinadora Contra la Represión Policial e Institucional; 2001.

21. Senado de la Nación. Respuesta enviada por el Ministerio de Justicia, Seguridad y Derechos Humanos, respecto de la fabricación, importación, exportación y comercialización de armas, así como aspectos relativos a los usuarios habilitados a operar con las mismas. Buenos Aires: Senado de la Nación; 2010.

22. Saín M. Seguridad, democracia y reforma policial en la Argentina. Buenos Aires: Fondo de Cultura Económica; 2002.

23. Arzadún D. Las instituciones policiales en la Argentina, el caso de la Policía Bonaerense y los prolegómenos de la reforma. In: Kaminsky G, editor. Tiempos inclementes. Culturas policiales y seguridad ciudadana. Remedios de Escalada: Ediciones de la UNLa; 2005. (Colección Planificación y Políticas Públicas, Serie Seguridad Ciudadana). p. 127-40.

24. Saín M. Situación del seguridad pública en la Argentina. Análisis de coyuntura y prospectiva. In: Alvárez A, Bertranou J, Fernández Pedemonte D, editores. Estado, democracia y seguridad ciudadana. Aportes para el debate. Buenos Aires: Programa de Naciones Unidas para el Desarrollo; 2008. p. 61-106.

25. Fleitas D. Las políticas de control de armas de fuego en la Argentina durante los años 2006 y 2007. Buenos Aires: Asociación para Políticas Públicas; 2007.

26. Spinelli H, Alazraqui M, Zunino G, Olaeta H, Poggese $\mathrm{H}$, Concaro $\mathrm{C}$, et al. Firearm-related deaths and crime in the Autonomous City of Buenos Aires, 2002. Ciênc. Saúde Coletiva 2006; 11:327-38.

27. Kosovsky D. Las armas de fuego y los dos demonios en la seguridad pública. In: Alvárez A, Bertranou J, Fernandez Pedemonte D, editores. Estado, democracia y seguridad ciudadana. Aportes para el debate. Buenos Aires: Programa de las Naciones Unidas para el Desarrollo; 2008. p. 267-99.

Recibido el 04/Ene/2011

Versión final presentada el 03/Nov/2011

Aprobado el 05/Ene/2012 